\title{
MULTI-WAVELENGTH EMISSIONS FROM THE MILLISECOND PULSAR BINARY PSR J1023+0038 DURING AN ACCRETION ACTIVE STATE
}

\author{
J. Takata ${ }^{1}$, K. L. Li $^{2}$, Gene C. K. Leung ${ }^{1}$, A. K. H. Kong ${ }^{2}$, P. H. T. Tam ${ }^{2}$, C. Y. Hui ${ }^{3}$, E. M. H. Wu ${ }^{1}$,

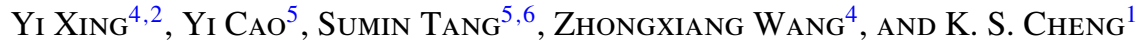 \\ ${ }^{1}$ Department of Physics, University of Hong Kong, Pokfulam Road, Hong Kong, China; takata@ hku.hk \\ ${ }^{2}$ Institute of Astronomy and Department of Physics, National Tsing Hua University, Hsinchu 30013, Taiwan; akong@ phys.nthu.edu.tw \\ ${ }^{3}$ Department of Astronomy and Space Science, Chungnam National University, Daejeon, Republic of Korea \\ ${ }^{4}$ Shanghai Astronomical Observatory, Chinese Academy of Sciences, 80 Nandan Road, Shanghai 200030, China \\ ${ }^{5}$ Astronomy Department, California Institute of Technology, 1200 E. California Boulevard, Pasadena, CA 91125, USA \\ ${ }^{6}$ Kavli Institute for Theoretical Physics, University of California, Santa Barbara, CA 93106, USA \\ Received 2013 November 30; accepted 2014 March 1; published 2014 April 3
}

\begin{abstract}
Recent observations strongly suggest that the millisecond pulsar binary PSR J1023+0038 has developed an accretion disk since 2013 June. We present a multi-wavelength analysis of PSR J1023+0038, which reveals that (1) its gammarays suddenly brightened within a few days in 2013 June/July and has remained at a high gamma-ray state for several months; (2) both UV and X-ray fluxes have increased by roughly an order of magnitude; and (3) the spectral energy distribution has changed significantly after the gamma-ray sudden flux change. Time variabilities associated with UV and X-rays are on the order of 100-500 s and 50-100 s, respectively. Our model suggests that a newly formed accretion disk, due to the sudden increase of the stellar wind, could explain the changes of all these observed features. The increase of UV is emitted from the disk, and a new component in gamma-rays is produced by inverse Compton scattering between the new UV component and pulsar wind. The increase of X-rays results from the enhancement of injection pulsar wind energy into the intra-binary shock due to the increase of the stellar wind. We also predict that the radio pulses may be blocked by the evaporated winds from the disk, and the pulsar is still powered by rotation.
\end{abstract}

Key words: accretion, accretion disks - binaries: close - pulsars: individual (PSR J1023+0038)

Online-only material: color figure

\section{INTRODUCTION}

The formation and evolution of millisecond pulsars (MSPs) have been a subject of debate. It is widely believed that neutron stars in low-mass X-ray binaries (LMXBs) can be spun up to millisecond periods by gaining angular momentum from the accreting materials of the companion star (e.g., Alpar et al. 1982). When the accretion stops, the neutron stars become radio MSPs powered by rotation (e.g., Campana et al. 1998). The first direct observational evidence to support the link between MSPs and LMXBs comes from the discovery of the X-ray binary/MSP PSR J1023+0038 (J1023 hereafter). J1023 was first identified as an LMXB with an orbital period of $4.75 \mathrm{hr}$ based on its X-ray and optical properties (Woudt et al. 2004; Thorstensen \& Armstrong 2005; Homer et al. 2006) and the millisecond pulsation $(P=1.69 \mathrm{~ms})$ was subsequently found (Archibald et al. 2009). The source clearly showed an accretion disk between 2000 and 2001 (Wang et al. 2009), and the disk has since disappeared (Archibald et al. 2009) while radio pulsation was found in 2007, confirming the MSP nature (Archibald et al. 2009). Therefore, J1023 is considered as a newly born MSP, representing the missing link of a rotation-powered MSP descended from an LMXB. In such a system, that recently transited from the LMXB phase to the radio MSP phase, it is widely believed that the accretion disk of J1023 will reform due to an increase in mass transfer rate, and the radio MSP will then be switched off (Archibald et al. 2009; Wang et al. 2009; Tam et al. 2010). One very recent example is IGR J18245-2452 in the globular cluster M28, which has been switching between an accretion and rotation-powered MSP (Papitto et al. 2013).
Since late June 2013, the radio pulsation of J1023 has disappeared between $350 \mathrm{MHz}$ and $5 \mathrm{GHz}$ (Stappers et al. 2013; Patruno et al. 2014). Meanwhile, recent optical spectroscopy shows strong double-peaked $\mathrm{H} \alpha$ emission, indicating that an accretion disk has formed (Halpern et al. 2013). Moreover, the X-ray emission has increased by a factor of at least 20 comparing with previous quiescent values (Kong 2013), and the UV emission has brightened by 4 mag (Patruno et al. 2014). All of these strongly indicate that J1023 is switching from a radio MSP to an LMXB with an accretion disk. In addition, the gamma-ray emission ( $>100 \mathrm{MeV}$ ), as seen with Fermi, has been reported to brighten by a factor of five (Stappers et al. 2013; Patruno et al. 2014). It is somewhat unusual since we expect that the gamma-rays should be turned off like radio during the accretion state (e.g., Takata et al. 2010, 2012). In fact, no LMXB has been detected by Fermi so far.

In this paper, we report a multi-wavelength (from optical to gamma-ray) study of J1023 during its recent accretion active state (Section 2), and propose a model to explain the multiwavelength behaviors (Section 3). A brief summary and discussion on observed variabilities in multi-wavelength emissions are given in Section 4.

\section{SUMMARY OF MULTI-WAVELENGTH OBSERVATIONS}

\subsection{Fermi Large Area Telescope $\gamma$-Ray Observations}

Gamma-ray data were obtained, reduced, and analyzed using the Fermi Science Tools package (v9r32p5), available 
from the Fermi Science Support Center. ${ }^{7}$ We selected events in the reprocessed Pass 7 "Source" class and used the P7REP_SOURCE_V15 version of the instrumental response functions. To reduce contamination from the Earth's albedo, we excluded time intervals when the region of interest (ROI) was observed at zenith angles greater than $100^{\circ}$ or when the rocking angle of the Large Area Telescope (LAT) was greater than $52^{\circ}$. We used photons between 0.1 and $300 \mathrm{GeV}$ within a $20^{\circ} \times 20^{\circ}$ ROI centered at the position of J1023. The Fermi LAT was under the sky survey mode for most of the time considered, allowing the full sky to be surveyed every three hours.

We performed binned likelihood analyses with the gtlike tool. For source modeling, all 2FGL catalog sources (Nolan et al. 2012) within $19^{\circ}$ of the ROI center, the galactic diffuse emission (gll_iem_v05.fits), and isotropic diffuse emission (iso_source_v05.txt) were included. For sources more than $10^{\circ}$ away from the position of $\mathrm{J} 1023$, the spectral parameters were fixed to the catalog values.

\subsubsection{Spectral Analysis}

We used Fermi LAT data collected in two separate time intervals: from 2008 August 14 to 2013 May 31 and from 2013 July 1 to 2013 November 12, roughly corresponding to the time before and after the $\gamma$-ray state change (see below for a temporal analysis), respectively. In each of the two time intervals, we performed two separate fits. We modeled J1023 with a simple power law

$$
\frac{d N}{d E}=N_{0}\left(\frac{E}{E_{0}}\right)^{-\Gamma},
$$

as well as a power law with exponential cutoff (PLE)

$$
\frac{d N}{d E}=N_{0}\left(\frac{E}{E_{0}}\right)^{-\Gamma} \exp \left(-\frac{E}{E_{c}}\right) .
$$

Before the state change, the fit with a simple power law gives $\Gamma=2.6 \pm 0.2$ and $F_{\gamma}=(9.5 \pm 3.0) \times 10^{-9}$ photons cm $\mathrm{cm}^{-2} \mathrm{~s}^{-1}$. The fit with a PLE gives $\Gamma=1.4 \pm 0.6, E_{c}=0.7 \pm 0.4 \mathrm{GeV}$ and $F_{\gamma}=(7.4 \pm 2.4) \times 10^{-9}$ photons $\mathrm{cm}^{-2} \mathrm{~s}^{-1}$. These spectral parameters are consistent with those first reported in Tam et al. (2010). The likelihood ratio test gives $2 \Delta \log$ (likelihood) $\approx 8.8$, indicating that the improvement by the PLE model has a significance of just below $3 \sigma$, noting that the PLE model has one more degree of freedom. Using $1.5 \mathrm{yr}$ of data, Tam et al. (2010) found that the two models fit the data equally well, due to the lower photon statistics.

After the state change, the best-fit parameters for the simple power law are $\Gamma=2.3 \pm 0.1$ and $F_{\gamma}=(8.9 \pm 1.1) \times$ $10^{-8}$ photons $\mathrm{cm}^{-2} \mathrm{~s}^{-1}$. The best-fit parameters for the PLE model are $\Gamma=1.8 \pm 0.2, E_{c}=2.3 \pm 0.9 \mathrm{GeV}$ and $F_{\gamma}=$ $(7.4 \pm 1.0) \times 10^{-8}$ photons $\mathrm{cm}^{-2} \mathrm{~s}^{-1}$. The likelihood ratio test gives $2 \Delta \log ($ likelihood $) \approx 8.8$, indicating that the improvement by the PLE model has a significance of slightly below $3 \sigma$. There is approximately a factor of 10 increase in the photon flux after the state change, confirming an earlier report (Stappers et al. 2013).

Spectral points were obtained by fitting the normalization factors of point sources within $6^{\circ}$ from the pulsar and the diffuse backgrounds in individual energy bins. Other parameters were fixed to the best-fit values obtained in the full energy band analysis. The $95 \%$ c.l. upper limits were calculated in energy bins where $\mathrm{J} 1023$ had a significance below $3 \sigma$.

\footnotetext{
http://fermi.gsfc.nasa.gov/ssc/data/analysis/software/
}

\subsubsection{Temporal Analysis}

To show the change in $\gamma$-ray flux just before and after the state change, we constructed $\gamma$-ray light curves since 2013 June, as shown in Figure 1, with the one shown in the inset focusing on the time interval of the $\gamma$-ray state change seen in late June/ early July. The same source model used in the aforementioned spectral analysis was used in deriving each flux value in the light curves, except that the power law model was assumed for J1023 due to the shorter time scales. The bin size is 14 days and 3 days for the main panel and inset, respectively. Upper limits were derived for those time intervals in which the test-statistic (TS) values were below 9 (4) for the main panel (inset), assuming a power law index of $\Gamma=2.3$. It is clear that $\mathrm{J} 1023$ cannot be detected in 2 weeks time before 2013 July; we designate such time interval as low $\gamma$-ray state. After the state change (a time interval we call high $\gamma$-ray state), the $\gamma$-ray flux has remained at a much higher level for most of the time.

We estimated the dates of the state change, i.e., when the binary system changed from the low $\gamma$-ray state to the high $\gamma$-ray state, as follows. Likelihood analyses were performed for the time intervals spanning 1 week ( 7 days). The first time interval that we used starts at June 20 and ends June 26; then we shifted the analyzed time interval for 1 day, i.e., the next time interval is hence June $21-27$, the third time interval is June $22-28$, and so on. The last time interval is July 8-14.

In the beginning, TS values are below 10 . The first bin, having TS $>10$, occurred at June 29-July 5. Note that never before (or, never since 2013 March) could J1023 be detected at this significance with only 1 week of data, so during this particular week (June 29-July 5), J1023 has undergone a state change from the low state to high state in $\gamma$-rays. We indicate this time interval in Figure 1.

To look for orbital modulation, we mapped the orbital phase to the geocentric arrival time and divided the data into two halves in the orbital phase, each centered at one of the conjunctions. We performed the same analysis as above in each phase interval and did not detect any significant orbital modulation.

\subsection{XRT Observations}

Since the disappearance of the radio pulsation of J1023, the pulsar has been monitored by Swift/X-Ray Telescope (XRT) for the X-ray evolution. The X-ray properties observed on 2013 June 10 and 12 (Kong 2013) are roughly consistent with previous XMM-Newton observations taken in 2004 and 2008 (Archibald et al. 2010; Tam et al. 2010), indicating that the system was still in quiescence. Since October 18, an enhanced X-ray emission was detected from a 10 ks XRT observation (Kong 2013; Patruno et al. 2014), reflecting rapid X-ray activity in the binary system. Since then, a daily monitoring campaign was triggered to monitor J1023 almost daily in November (Papitto et al. 2013), and the X-ray source has stayed at a high flux level with high variabilities on timescales of a few tens of seconds. In this study, we mainly focus on the observations taken after the Xray brightening (i.e., October 18) to investigate the origin of the enhanced X-ray radiations. All of the Swift observations used in this analysis are listed in Table 1.

We downloaded the observations from the Swift Quick Look Archive and extracted spectra as well as lightcurves by the HEAsoft (ver. 6.14) Swift-specific tasks, xrtgrblc and xrtgrblcspec, in which count-rate-dependent regions are applied. As no significant spectral variability are seen among the individual observations during the high state, we combined all 


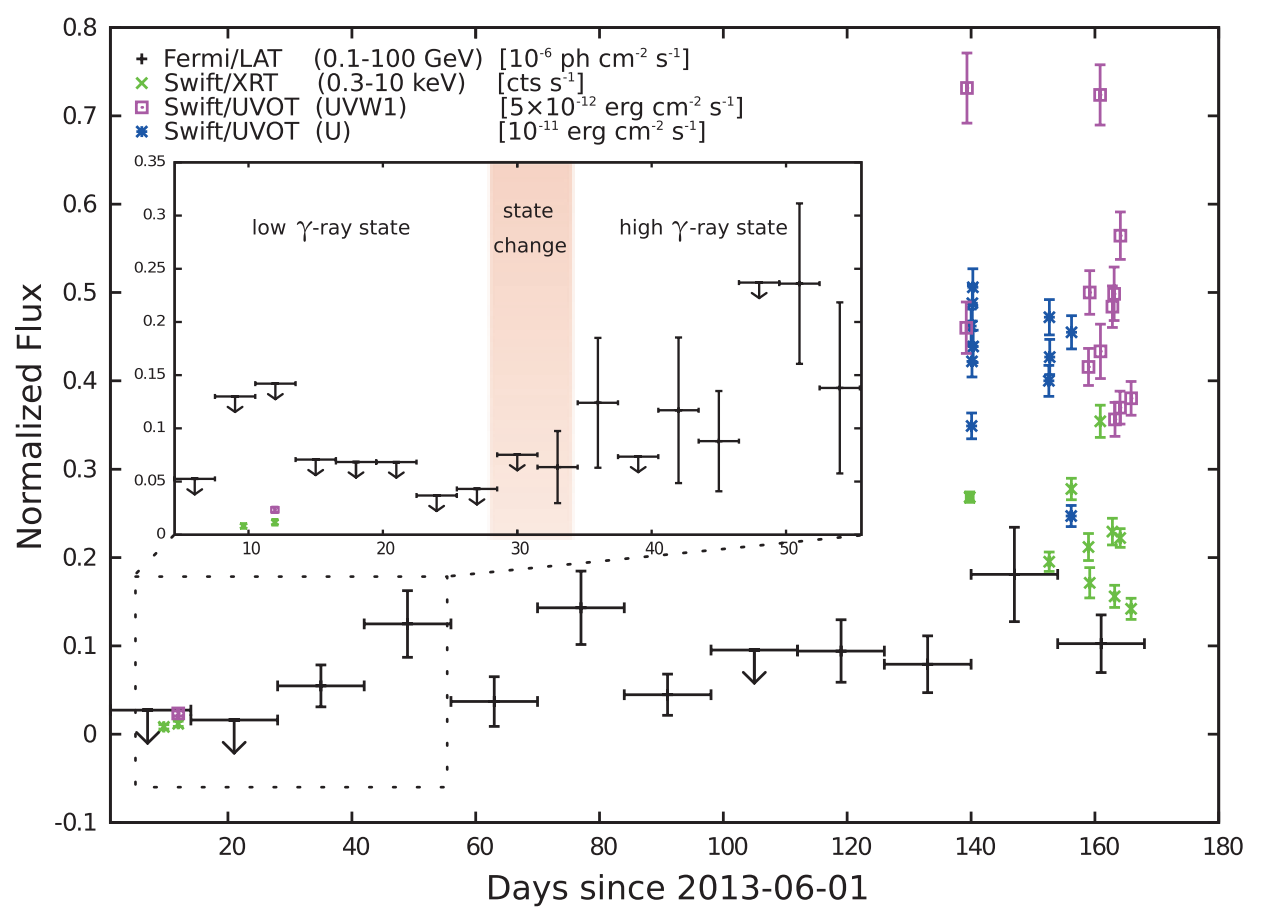

Figure 1. Main panel shows the multi-wavelength (i.e., from UV to $\gamma$-ray) lightcurves of PSR J1023+0038 from June 1 to November 13 with different flux scales for each energy band (see the upper left corner for details) while the inset box indicates the detailed evolution of the $\gamma$-ray emissions between June 6 to 24 July. UV/X-ray: each datum represents an individual observation taken by Swift. $\gamma$-ray: each datum in the main panel (inset) corresponds to 2 weeks (3 days), and $95 \%$ c.l. upper limits are given for the time intervals during which test-statistic (TS) values were below 9 (4).

(A color version of this figure is available in the online journal.)

Table 1

Swift Observations of PSR J1023+0038

\begin{tabular}{llcccc}
\hline \hline Obs Id & $\begin{array}{c}\text { Date }^{\mathrm{a}} \\
(\mathrm{m} / \mathrm{d})\end{array}$ & $\begin{array}{c}\text { Exp } \\
(\mathrm{s})\end{array}$ & $\begin{array}{c}\text { UVOT filter } \\
(U / U V W 1)\end{array}$ & $\begin{array}{c}\text { Mode }^{\mathrm{b}} \\
(\mathrm{I} / \mathrm{E})\end{array}$ & $\begin{array}{c}\text { X-ray state } \\
(\text { High } / \text { Low })\end{array}$ \\
\hline 80035001 & Jun 10 & 1918 & $U V W 2$ & $\mathrm{I}$ & $\mathrm{L}$ \\
80035002 & Jun 12 & 1863 & $U V W 1$ & $\mathrm{I}$ & $\mathrm{L}$ \\
80035003 & Oct 18/19 & 9907 & $U$ and $U V W 1$ & $\mathrm{I}$ & $\mathrm{H}$ \\
33012001 & Oct 31 & 1910 & $U$ & $\mathrm{I}$ & $\mathrm{H}$ \\
33012002 & Nov 04 & 2083 & $U$ & $\mathrm{I}$ & $\mathrm{H}$ \\
33012003 & Nov 06 & 1011 & $U V W 1$ & $\mathrm{E}$ & $\mathrm{H}$ \\
33012004 & Nov 07 & 939 & $U V W 1$ & $\mathrm{E}$ & $\mathrm{H}$ \\
33012005 & Nov 08 & 1264 & $U V W 1$ & $\mathrm{E}$ & $\mathrm{H}$ \\
33012007 & Nov 10 & 1116 & $U V W 1$ & $\mathrm{E}$ & $\mathrm{H}$ \\
33012008 & Nov 11 & 1134 & $U V W 1$ & $\mathrm{E}$ & $\mathrm{H}$ \\
33012009 & Nov 12 & 2295 & $U V W 1$ & $\mathrm{E}$ & $\mathrm{H}$ \\
33012010 & Nov 13 & 1121 & $U V W 1$ & $\mathrm{E}$ & $\mathrm{H}$ \\
\hline
\end{tabular}

Notes.

a All observations were taken in 2013.

b UVOT observing mode. I: IMAGE mode; E: EVENT mode.

of the selected events to construct an averaged X-ray spectrum $(0.3-10 \mathrm{keV})$ with a total exposure time of $22.6 \mathrm{ks}$, which can be well described by an absorbed power law model with best-fit parameters of $N_{\mathrm{H}}=5.2_{-1.5}^{+1.6} \times 10^{20} \mathrm{~cm}^{-2}$ and $\Gamma=1.76_{-0.06}^{+0.07}\left(\chi_{v}^{2}=\right.$ 0.95 with dof $=170)$, inferring an observed flux of $F_{0.3-10}=$ $(9.8 \pm 0.4) \times 10^{-12} \mathrm{erg} \mathrm{cm}^{-2} \mathrm{~s}^{-1}$ and an absorption corrected luminosity of $L_{0.3-10}=(2.20 \pm 0.07) \times 10^{33}(d / 1.3 \mathrm{kpc})^{2} \mathrm{erg} \mathrm{s}^{-1}$. Comparing with previous XMM-Newton results (Tam et al. 2010), the X-ray luminosity is increased by at least a factor of 20 with a much softer photon index. We also tried to improve the fit by adding a thermal model, such as bbody and mekal (XSPEC built-in models). Although it slightly improves the chi-square statistic, the $F$-test indicates the null-hypothesis probabilities for the bbody and mekal cases to be $14 \%$ and $54 \%$, respectively, suggesting that the improvement is not significant. In addition, the featureless X-ray spectrum does not favor any plasma emission contributions.

As the average observed count rate is about 0.21 counts $\mathrm{s}^{-1}$, a $50 \mathrm{~s}$ bin time is used for the lightcurve binning to achieve a mean signal-to-noise ratio of approximately $>3$ per bin. Shortterm variabilities on timescales of a few tens of seconds are clearly seen in the curve with variations up to a factor of 10 between two consecutive bins. Interestingly enough, at least 21 of the data bins (with fractional exposure equals one) have a zero count rate, which are unlikely random events due to an extremely low Poisson probability of getting a null count in a $50 \mathrm{~s}$ exposure (i.e., $0.0027 \%$ ). Some of the zero count bins are close enough with other low count rate bins $(\leqslant 0.07$ counts $\mathrm{s}^{-1}$ ) to form several low-flux intervals in ranges of 200-550 s. Meanwhile, the peak flux reaches $1.64 \pm 0.23$ counts s $^{-1}$, which arises from $0.10 \pm 0.06$ counts s$^{-1}$ in $100 \mathrm{~s}$, stays for about $50 \mathrm{~s}$, and then drops to $0.06 \pm 0.05$ counts $\mathrm{s}^{-1}$ in $100 \mathrm{~s}$. However, no significant hardness variability is found despite the highflux variability. The HEAsoft tasks, powspec and ef search, were employed to search for the signals from the known orbital and pulsar periods (Archibald et al. 2009; Wang et al. 2009). Although no reliable signal regarding the orbit or pulsation is found, an unknown periodic signal of $P \approx 245 \mathrm{~s}$ is detected from UT 6:36:42 to 7:04:35 on October 19 (about seven cycles), which is not detected in other observations.

\subsection{UVOT Observations}

During the X-ray high state, Swift/UVOT observed the source simultaneously with the XRT using the $U(3465 \AA)$ and/or $U V W 1(2600 \AA)$ band filters with IMAGE or EVENT mode (Table 1). The optical/UV counterpart is clearly detected in 
all UVOT images. Using the HEAsoft tasks, uvotmaghist and uvotevtlc, we found that the UV magnitudes are much brighter than those quiescent measures taken in June by $\sim 3.7 \mathrm{mag}$. The UV emission varies among the observations between 16.76-15.98 mag $(U)$ and 16.90-16.12 mag (UVW1) with uncertainties less than $0.07 \mathrm{mag}$ (extinction uncorrected). No trend can be found when folding the obtained lightcurve by the orbital period $P_{\text {obs }}=0.198094$ (2) days (Thorstensen \& Armstrong 2005), suggesting that the variability is not orbit related. EVENT mode observations were started from November 6 , which allowed us to investigate short-term variabilities with timescales down to a few tens of seconds. We chose a binning factor of $50 \mathrm{~s}$ and found that the binned lightcurves are full of variabilities with timescales between $\sim 100$ and $\sim 500 \mathrm{~s}$. The corresponding XRT data were examined but no clear correlation between the UV lights and the X-rays was found.

\subsection{Keck/DEIMOS Optical Spectrum}

We obtained a $500 \mathrm{~s}$ spectroscopic observation from the DEIMOS multi-object spectrograph with a 0.8 arcsec slit on the Keck II telescope at UT 15:29:15 on November 3. The spectrum covers from $4750 \AA$ to $9600 \AA$ at $2.8 \AA$ resolution. The DEEP2 DEIMOS Data Pipeline was used for the CCD processing and flux was calibrated using HD19445 and the last photometric measure of Halpern et al. (2013; i.e., $V=$ $16.44 \mathrm{mag}$ ). Due to an unstable weather condition during the observation, as well as the large spectral deviation between the calibrator (Ap) and the target (G-type+low temperature accretion disk), we note that the flux calibration may not be very accurate. Obvious emission lines of $\mathrm{H} \alpha$, $\mathrm{He}$ I $\lambda 5876$, and He I $\lambda 6678$ are detected in the spectrum and a double-peak feature is marginally seen in the $\mathrm{H} \alpha$ line profile with a peak separation of $\sim 19 \AA$, equivalent to a radial velocity difference of $\sim 860 \mathrm{~km} \mathrm{~s}^{-1}$, which is consistent with the earlier spectroscopic analysis from Halpern et al. (2013). Similar to the Sloan Digital Sky Survey (SDSS) spectrum taken during the last accretion state in 2000-2001 (Wang et al. 2009), the continuum can be well fitted by a multi-temperature disk model plus an irradiated G5V companion of $17.35 \mathrm{mag}$, given that the observation was taken during the plateau of the companion's modulated light curve (Thorstensen \& Armstrong 2005; Wang et al. 2009). Detailed accretion disk modeling will be discussed in the theory section (Section 3).

\subsection{Lulin One-meter Telescope}

On November 16, we observed J1023 with the Lulin Onemeter Telescope (LOT) at Lulin Observatory in Taiwan, using SDSS $g, r$, and $i$ filters. Two $g$-band ( 1 minute and 2 minutes), six $r$-band (2 minutes), plus one $i$-band (2 minutes) exposures were obtained under a good weather condition. Standard data reduction and aperture photometry were performed by the IRAF packages to compute the mean magnitudes $(g=17.16 \pm 0.02 \mathrm{mag}, r=16.63 \pm 0.01 \mathrm{mag}$, and $i=16.42 \pm 0.03 \mathrm{mag}$ ) for which the flux calibration was done by using three SDSS DR8 bright sources in the field (i.e., SDSS J102354.69+003516.2, J102357.97+004027.7, and J102338.46+003624.4), and the errors were calculated by assuming a Poisson distribution with standard propagation of errors. The photometry is roughly consistent but less blue than the DEIMOS spectrum. For the $r$-band images, we took the first image at 19:30 UT (near the time of inferior conjunction; see Thorstensen \& Armstrong 2005 for details) and consecutively made the rest of the observations (every $140 \mathrm{~s}$ ) 36 minutes later. A large magnitude change of $\Delta m=0.15 \pm 0.03$ is seen from the first two images and small variabilities up to $\Delta m=0.09 \pm 0.02$ in $140 \mathrm{~s}$ are shown in the consecutive data set. For the $g$-band magnitudes, we transformed them from an SDSS system to a Johnson filter system (Jester et al. 2005) and compared them with the ones reported in Halpern et al. (2013). The transformed $V$ magnitudes $(16.95 \pm 0.02$ and $16.88 \pm 0.02)$ are very close to Halpern's values at a similar orbital phase.

\subsection{Radio Observations}

The radio emission from the position of $\mathrm{J} 1023$ was first reported by the FIRST Very Large Array (VLA) $1.4 \mathrm{GHz}$ survey (Becker et al. 1995). In re-examining the FIRST data, Bond et al. (2002) realized that the detection reported by Becker et al. (1995) was based on a single $\sim 6.6 \mathrm{mJy}$ flare from a 1.4 GHz observation on 1998 August 10. On the other hand, the observations on 1998 August 3 and 1998 August 8 resulted in nondetections, and the corresponding limiting flux densities are $<1.8 \mathrm{mJy}$ and $<3.4 \mathrm{mJy}$, respectively (Bond et al. 2002). These observations clearly demonstrate the variability of the radio emission from this binary system, which can vary by a factor of $\sim 4$ over a time interval as short as a week. However, without the optical spectroscopic results from this epoch, it remains uncertain whether the system was in a rotation-powered or an accretion-powered state. But transient radio emission from a neutron star in an accreting binary is possible (Gaensler et al. 1999). During the rotation-powered phase, the radio emission from J1023 becomes more intense. Archibald et al. (2009) reported a mean flux density of $\sim 14 \mathrm{mJy}$ at $1.6 \mathrm{GHz}$. Recently, a long-term radio monitoring campaign of $\mathrm{J} 1023$ from mid2008 to mid-2012 reports the variable radio properties such as variable eclipses, short-term disappearance of signal, and excess dispersion measure at random orbital phases (Archibald et al. 2013).

Based on a radio observation at $1.4 \mathrm{GHz}$ performed on 2013 June 23, Stappers et al. (2013) reported the nondetection of pulsed radio emission from $\mathrm{J} 1023$ with a limiting flux density of $<0.06 \mathrm{mJy}$. However, one should be cautious in interpreting the result as it does not necessarily provide evidence for the quench of coherent radio emission mechanism. For an alternative scenario, assuming the pulsar remains active, the matter transferred from the companion toward the neutron star can be ejected by the pulsar wind (Ruderman et al. 1989). Also, the matter evaporated from the disk by the pulsar can further complicate the circumstellar environment. Therefore, even with the presence of an active pulsar mechanism, the nondetection of pulsed radio emission can possibly be due to the enhanced scattering/dispersion in the environment, which results in a smearing of the pulsed signal. Here, we build a theoretical model to explain the multi-wavelength observations of J1023 in its current accretion active state.

\section{THEORETICAL MODEL}

In this section, we discuss a possible scenario for the emission processes in multi-wavelength bands after late 2013 June. Figure 2 shows a schematic view of the system discussed in this section. With the observed flux of the UV emissions $\left(L_{\mathrm{UV}} \sim 10^{33} \mathrm{erg} \mathrm{s}^{-1}\right)$, the standard gas pressure-supported disk model (Frank et al. 2002) implies a mass loss rate of $\dot{M}_{16}=\dot{M} / 10^{16} \mathrm{~g} \mathrm{~s}^{-1} \sim 1$, and the inner edge of the accretion disk is $R_{\text {in }} \sim 10^{9-10} \mathrm{~cm}$ from the pulsar. This result suggests 


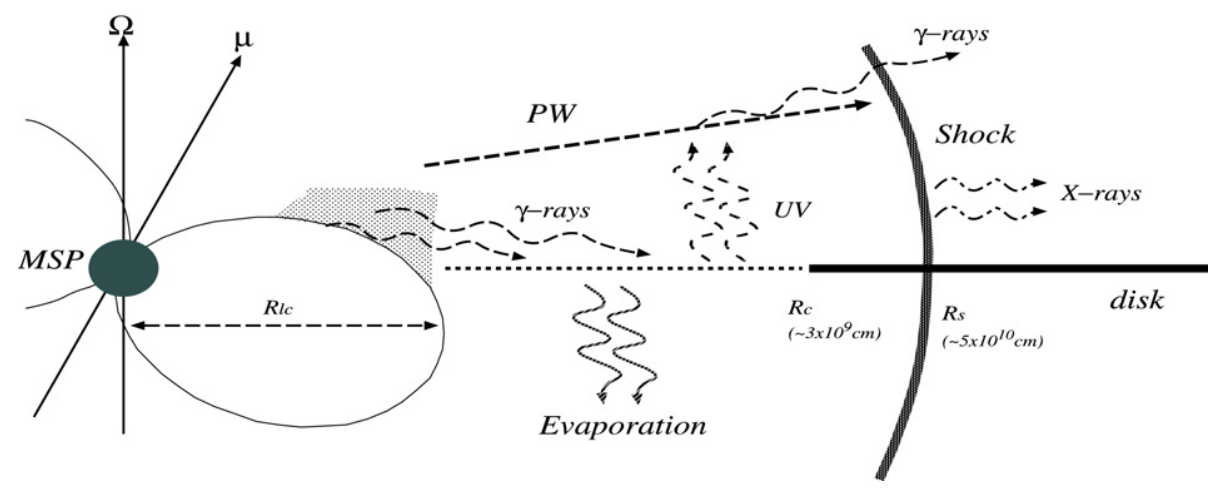

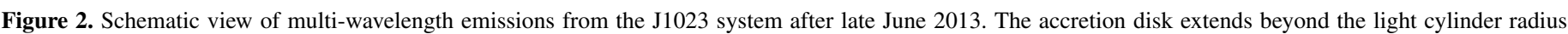

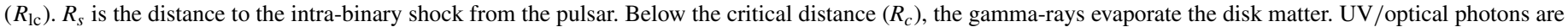

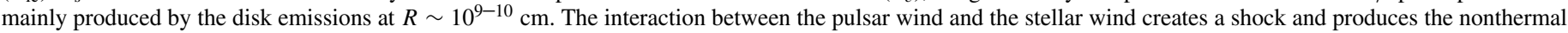
$\mathrm{X}$-ray emissions. The inverse Compton process of the cold relativistic pulsar wind off UV/optical photons from the disk produces the gamma-rays.

that the accretion disk does not substantially extend down to the pulsar or the emissions from the disk below $10^{9-10} \mathrm{~cm}$ are inefficient. Indeed, from our optical spectroscopy, the peak velocity of $\mathrm{H} \alpha$ infers an outer disk size of $\sim 10^{10} \mathrm{~cm}$. Takata et al. (2010) proposed that heating associated with deposition of the gamma-ray radiation from pulsar magnetosphere onto the accretion disk evaporates the accretion disk of an LMXB in quiescent state. The gamma-rays are absorbed via the socalled pair-creation process in a Coulomb field by nuclei (e.g., Liang 1999), if the disk column density exceeds $\Sigma_{c} \sim$ $10^{2} \mathrm{~g} \mathrm{~cm}^{-2}$. The energy conversion from the gamma-rays to the disk matter promotes the evaporation of the disk. Applying the standard disk model, the critical axial distance from the pulsar, below which the gamma-rays can evaporate the disk, is $R_{c} \sim 3 \times 10^{9} \alpha_{0.1}^{-16 / 15} \dot{M}_{16}^{14 / 15} \Sigma_{c, 2}^{-4 / 3} \mathrm{~cm}$, where $\alpha_{0.1}$ is the viscosity parameter in units of $0.1, \dot{M}_{16}$ is the accretion rate in units of $10^{16} \mathrm{~g} \mathrm{~s}^{-1}$, and $\Sigma_{c, 2}$ is the critical column density in units of $10^{2} \mathrm{~g} \mathrm{~cm}^{-2}$.

We expect that below the critical radius, the accretion disk will move toward the pulsar with a lower accretion rate. The mass loss from the disk due to the gamma-ray deposition reduces the rate of the viscous dissipation, and the distance variation of surface temperature of the disk is more moderate than the one $\left(T_{d} \propto R^{-3 / 4}\right)$ predicted by the standard disk model, in which the accretion rate is constant with the axial distance. Because the temperature distribution of the accretion disk evaporation is not obvious due to the irradiation of the gamma-rays, we assume a constant temperature at $T_{d}\left(R<R_{c}\right)=T_{d}\left(R_{c}\right)$ as the first order of approximation, where $T_{d}\left(R_{c}\right)$ is the temperature at the critical distance and is estimated by the standard disk model. Beyond the critical distance $R=R_{c}$, we apply the standard disk model, and we choose the temperature $T_{d}\left(R_{c}=10^{10} \mathrm{~cm}\right) \sim 8500 \mathrm{~K}$, which corresponds to an accretion rate of $\dot{M}_{16} \sim 1$, to explain the optical/UV data.

The existence of the GeV gamma-ray emissions even after the disappearance of the pulsed radio emission suggests that the accretion matter does not enter into the pulsar magnetosphere, and the rotation powered activities are still turned on. Hence, we assume that the disk extends beyond the light cylinder radius, $R_{\mathrm{lc}}=c P / 2 \pi \sim 8 \times 10^{6} \mathrm{~cm}$. The flux at the frequency $v$ from the disk is

$$
F_{v}=\frac{4 \pi h v^{3} \cos i}{c^{2} D^{2}} \int_{R_{\mathrm{lc}}}^{R_{\mathrm{out}}} \frac{R d R}{\exp ^{h v / k_{B} T(R)}-1}
$$

where $D \sim 1.3 \mathrm{kpc}$ is the distance to the system, $h$ is the Planck constant, $c$ is the speed of light, $k_{B}$ is the Boltzmann constant, and $i \sim 40^{\circ}-50^{\circ}$ (Wang et al. 2009) is the Earth viewing angle measured from the direction perpendicular to the orbital plane. With the present assumption of the temperature distribution, the luminosity of the disk emissions below $R=R_{c}$ is comparable to or smaller than that above $R=R_{c}$.

Tam et al. (2010) expected that the GeV emissions during the pulsed radio state originated from the outer gap in the pulsar magnetosphere because the inverse Compton processes of the shocked pulsar wind and the cold relativistic pulsar wind could not explain the observed flux level. The increase in the flux by a factor of 10 after the disappearance of the pulsed radio emissions implies that an additional component emerges and it dominates the magnetospheric component. We propose that the inverse Compton scattering process of the cold relativistic pulsar wind off the soft photons from the disk produces the additional gamma-rays. With the observed luminosity of the UV emissions from the disk, the depth of the inverse Compton scattering process is estimated to be of the order of $\tau_{i c} \sim n_{s} \sigma_{T} r \sim$ $0.4\left(L_{\mathrm{UV}} / 10^{33} \mathrm{erg} \mathrm{s}^{-1}\right)\left(E_{s} / 3 \mathrm{eV}\right)^{-1}\left(r / 10^{9} \mathrm{~cm}\right)^{-1}$, where $n_{s}=$ $L_{\mathrm{UV}} / 4 \pi r^{2} c E_{s}$ is the number density of the soft photons, $L_{\mathrm{UV}}$ is the luminosity of optical/UV emissions from the disk, $E_{s}$ is the soft photon energy, and $\sigma_{T}$ is the Thomson cross section. Hence, if the disk extends below $R \sim 10^{9} \mathrm{~cm}$, the inverse Compton of the cold relativistic pulsar wind can produce the observable gamma-rays. We note that the up-scattered photons will be observed as a steady component with the orbital phase because the accretion disk surrounds the pulsar.

It is believed that the cold relativistic pulsar wind forms near the light cylinder and carries almost all of the spin down energy into the interstellar space. We assume that the pulsar wind mainly consists of the electrons, positrons, and magnetic field, and that the energy distribution of the particles forms a monoenergetic function. The Lorentz factor $\left(\Gamma_{\mathrm{PW}}\right)$ of the bulk motion of the pulsar wind is related to the magnetization parameter $(\sigma)$, which is the ratio of the Poynting flux to the kinetic energy flux. Near the light cylinder, the magnetic energy dominates the particle energy, which is $\sigma \gg 1$. Beyond the light cylinder, the magnetic field may be dissipated so that the magnetic energy is converted into the particle energy of the flow (Coroniti 1990; Lyubarsky \& Kirk 2001). The Lorentz factor at the distance $R$ is estimated as

$$
\Gamma_{\mathrm{PW}}(R)=\left(\frac{1+\sigma_{L}}{1+\sigma(R)}\right) \Gamma_{\mathrm{PW}, L}
$$


where $\sigma_{L}$ and $\Gamma_{\mathrm{PW}, L}$ are the magnetization parameter and the Lorentz factor at the light cylinder, respectively. Because of the theoretical uncertainties of the evolution of the magnetization parameter beyond the light cylinder, we assume a power law form of

$$
\sigma(R)=\sigma_{L}\left(\frac{R}{R_{\mathrm{lc}}}\right)^{-\beta},
$$

where the index $\beta$ is the fitting parameter. In fitting the orbitaldependent spectrum of the gamma-ray binary PSR 1259-63/ LS2883, Kong et al. (2011, 2012) applied the above spatial dependent magnetization parameter to explain the observed data. We calculated the inverse Compton scattering process with an isotropic photon field,

$$
F\left(E_{\gamma}, R\right)=\int \frac{\sigma_{\mathrm{IC}}(R) c}{E_{s}} \frac{d N_{s}}{d E_{s}}(r) d E_{s},
$$

where $d N_{s} / d E_{s}$ is the soft photon field distribution from the disk, and the cross section $\sigma_{\text {IC }}$ is described by

$$
\begin{aligned}
\sigma_{\mathrm{IC}}(R)= & \frac{3 \sigma_{T}}{4 \Gamma_{\mathrm{PW}}^{2}(R)}[2 q \ln q+(1+2 q)(1-q) \\
& \left.+\frac{\left(\Gamma_{q} q\right)^{2}(1-q)}{2\left(1+\Gamma_{q} q\right)}\right]
\end{aligned}
$$

where $\Gamma_{q}=4 \Gamma_{\mathrm{PW}} E_{s} / m_{e} c^{2}, q=E_{0} / \Gamma_{q}\left(1-E_{0}\right)$ with $E_{0}=$ $E_{\gamma} / \Gamma_{\mathrm{PW}} m_{e} c^{2}$ and $1 /\left(4 \Gamma_{\mathrm{PW}}\right)^{2}<q<1$ (Blumenthal \& Gould 1970). The isotropic soft-photon field is a good approximation because the solid angle of the disk measured from the pulsar wind is not small, and the inverse Compton processes with various collision angles contribute to the emissions. We also take into account the anisotropic inverse Compton scattering process of the pulsar wind off the stellar photons, although its contribution to the emissions after late June 2013 is negligible.

After late June 2013, the X-ray emission does not show the orbital variation and increases by at least a factor of $\sim 20$. Previous X-ray observations during the radio MSP phase revealed orbital flux variation, implying the emissions from the intra-binary shock (Tam et al. 2010; Bogdanov et al. 2011). Bogdanov et al. (2011) suggested that the emission region near the companion star and its orbital variation are caused by the eclipse of the emission region by the companion star. The disappearance of the orbital variation after late June 2013 may suggest that the size of the emission region is bigger than that before late June 2013. We expect that the increase in the mass loss rate from the companion star pushes the emission region back toward the pulsar, and a greater fraction of the pulsar wind is stopped by the shock, resulting in an increase in the X-ray emissions from the system. The momentum ratio $(\eta)$ of the stellar wind and the pulsar wind is $\eta \sim$ $\left(\dot{M}_{w} v_{w} c / L_{\mathrm{sd}}\right) \sim 1\left(\dot{M}_{w} / 10^{16} \mathrm{~g} \mathrm{~s}^{-1}\right)\left(v_{w} / 10^{8} \mathrm{~cm} \mathrm{~s}^{-1}\right)\left(L_{\mathrm{sd}} / 5 \times\right.$ $\left.10^{34} \mathrm{erg} \mathrm{s}^{-1}\right)^{-1}$, indicating that the intrabinary shock stands at a distance $R_{s} \sim a / 2 \sim 5 \times 10^{10} \mathrm{~cm}$ from the pulsar if the mass loss rate of the stellar wind after late June 2013 increases to $\dot{M}_{w} \sim 10^{16} \mathrm{~g} \mathrm{~s}^{-1}$. With the momentum ratio $\eta$, the fraction $(\zeta)$ of the pulsar wind stopped by the shock is estimated as $\zeta \sim(1-\cos \psi) / 2$, where $\psi \sim 2.1\left(1-\eta^{2 / 5} / 4\right) \eta^{1 / 3}$ (Eichler \& Usov 1993). Before late June 2013, because we do not know the mass loss rate from the companion star, we assume that the $\mathrm{X}$-ray emission region is close to the L1-Lagrangian point, and the size of the emission region is approximately described by the size of the Roche-lobe. The solid angle of the Roche-lobe around the companion star measured from the pulsar is of the order of $\delta \Omega \sim \pi\left(R_{*} / a\right)^{2}$, where $R_{*} \sim 2 \times 10^{10} \mathrm{~cm}$ is the radius of the Roche-lobe and $a \sim 10^{11}$ is the separation between two stars. Hence, the fraction $(\zeta)$ of the pulsar wind stopped by the shock will increase from $\zeta \sim \delta \Omega / 4 \pi=1 \%$ before late June 2013 to several ten percents after late June 2013.

We expect that a strong shock forms if the magnetization parameter is smaller than or comparable to unity $\sigma\left(R_{S}\right) \leqslant 1$, and the shocked pulsar wind particles form a power law distribution described by $f(\gamma)=K \gamma^{-p}$. The maximum Lorentz factor $\left(\gamma_{\max }\right)$ is determined from the condition for which the particle acceleration time scale $t_{a c} \sim \gamma_{\max } m_{e} c / e B$ is equal to the synchrotron radiation loss timescale, $\tau_{\text {sync }} \sim 9 m_{e}^{3} c^{5} / e^{4} B \gamma_{\max }$, where $B=3\left[L_{\mathrm{sd}} \sigma\left(R_{s}\right) / R_{s}^{2} c\left(1+\sigma\left(R_{s}\right)\right)\right]^{1 / 2}$ is the magnetic field just behind the shock. The minimum Lorentz factor and the normalization factor are calculated from the two conditions, $\int f(\gamma) d \gamma=\zeta L_{\mathrm{sd}} /\left[4 \pi \Gamma_{\mathrm{PW}}\left(r_{s}\right) r_{s}^{2} m_{e} c^{3}\left(1+\sigma\left(r_{s}\right)\right)\right]$ and $\int \gamma f(\gamma) d \gamma=\zeta L_{\mathrm{sd}} /\left[4 \pi r_{s}^{2} c\left(1+\sigma\left(r_{s}\right)\right)\right]$, where $r_{s}$ is the radial distance to the shock from the pulsar. With a simple onedimensional model, we solve the distribution of the pulsar wind particles with the cooling processes via adiabatic expansion and radiation losses. We calculate the synchrotron radiation and the inverse Compton radiation process from the shocked pulsar wind.

Figure 3 summarizes the results of the model fitting for the observed emissions before (left panel) and after (right panel) late June 2013. The dotted, dashed, and dashed-dotted lines are the calculated spectra for the emissions from the outer gap (see Wang et al. 2010 for a detailed calculation), shocked pulsar wind, and the cold relativistic pulsar wind, respectively. The double-dotted line in the right panel shows the predicted spectrum of optical/UV emissions with the current model of the accretion disk. The flux predicted by the inverse Compton process of the cold relativistic pulsar wind before late June 2013 is of the order of $\sim 10^{-13} \mathrm{erg} \mathrm{cm}^{-2} \mathrm{~s}^{-1}$, and its spectrum is not shown in the figure. As we can see in the figure, the observed $\mathrm{GeV}$ emissions after late June 2013 can be explained well by the inverse Compton scattering process of the cold relativistic pulsar wind off the soft photons from the disk. We choose the parameters of the cold relativistic pulsar wind described by Equations (4) and (5) as $\sigma_{L} \sim 100, \Gamma_{\mathrm{PW}, L} \sim 200$ and the index $\beta \sim 0.7$, respectively. The observed flux level of the X-ray emissions after late June 2013 is also reproduced by the shock emissions with a shock distance of $R_{s} \sim 5 \times 10^{10} \mathrm{~cm}$ and a power law index of $p \sim 2.2$. Before late June 2013, a harder energy distribution $(p=1.6)$ of the particles at the shock is required to explain the observed photon index $(\sim 1.4)$ of the $\mathrm{X}$-ray emissions.

The matter evaporated from the disk will smear out the pulsed radio emissions from the neutron star via scattering and/or absorption, if the local plasma frequency is greater than the frequency of the radio wave. Assuming that the disk matter below $R_{c} \sim 3 \times 10^{9} \mathrm{~cm}$ are evaporated by the gamma-rays, one may estimate the local plasma frequency at the scattering region as $\left(\omega_{p} / 2 \pi\right) \sim$ $6 \mathrm{GHz}\left(\dot{M}_{d} / 10^{16} \mathrm{~g} \mathrm{~s}^{-1}\right)^{1 / 2}\left(v_{d, w} / 10^{8} \mathrm{~cm} \mathrm{~s}^{-1}\right)^{-1}\left(R_{c} / 3 \times 10^{9} \mathrm{~cm}\right)^{-1}$, where $\dot{M}_{d}$ is the evaporation of the disk matter, and $v_{d, w}$ is the velocity of the evaporated matter. This suggests that the matter evaporated from the disk smears out the pulsed radio emissions up to a frequency of several $\mathrm{GHz}$, which is consistent with 

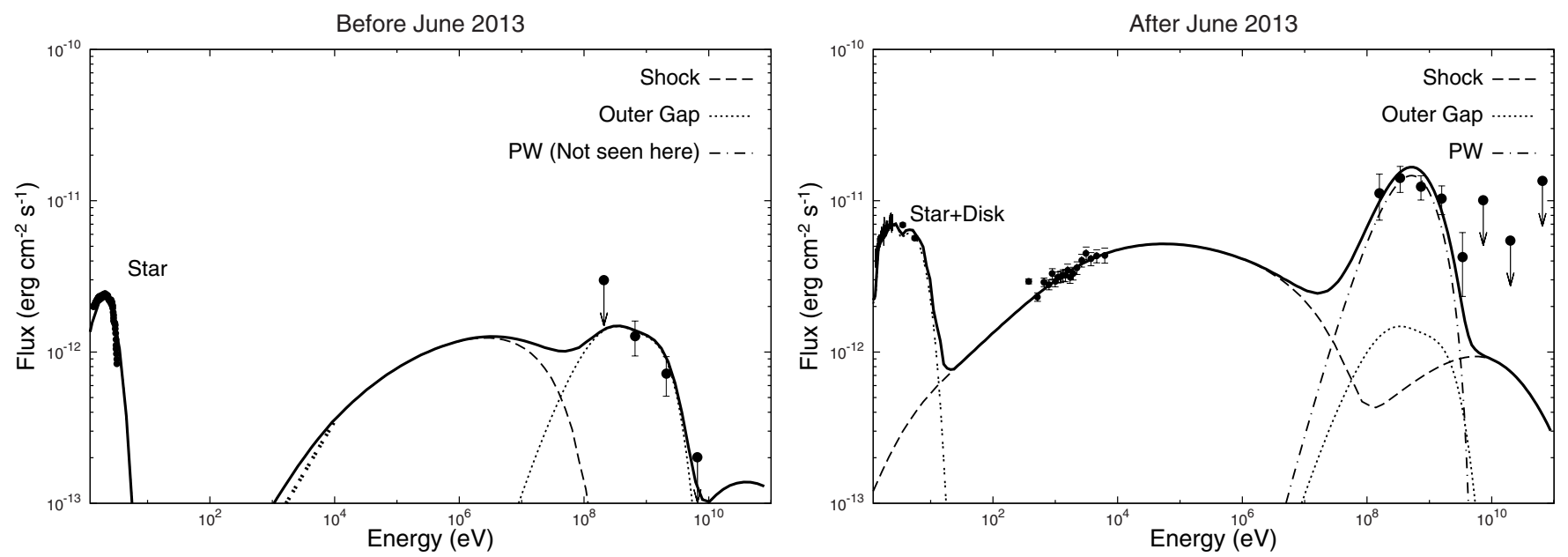

Figure 3. Multi-wavelength spectra before (left) and after (right) late June 2013. The dashed, dotted, and dashed-dotted line represent the calculated spectra of the emissions from the shock, outer gap (see Wang et al. 2010), and cold relativistic pulsar wind, respectively. For the shock emissions, we assume a distance of $R_{s} \sim 8 \times 10^{10} \mathrm{~cm}$ and a power law index of $p=1.6$ before late June 2013 and $R_{s} \sim 5 \times 10^{10} \mathrm{~cm}$ and $p=2.2$ after late June 2013, respectively. In the left panel, the stellar (G5V) spectrum (thick-curve line) and X-ray data (thick-double-dotted line) are taken from Wang et al. (2009) and Tam et al. (2010), respectively. The flux predicted by inverse Compton process of the cold relativistic pulsar wind before late June 2013 is of the order of $\sim 10^{-13} \mathrm{erg} \mathrm{cm}^{-2} \mathrm{~s}^{-1}$, and its spectrum is not seen in the figure.

observations that no radio pulsation has been detected below $5 \mathrm{GHz}$ during the accretion active state. In this scenario, the rotation-powered MSP is still active. While detecting the pulsation can be difficult, radio imaging can still be used to check the aforementioned scenario as such a technique is not hampered by the complicated environment. Also, as the local charge density can possibly be increased by the aforementioned processes, if the observed frequency is below the local plasma density, this can lead to a nondetection, even with imaging. In view of this, a high-frequency radio imaging observation is encouraged to confirm/refute this alternative scenario.

\section{DISCUSSION}

We performed a multi-wavelength study of the MSP binary J1023 using UV/optical, X-ray, and gamma-ray data during its accretion active state starting in late 2013 June. Not only are there significant UV/optical and X-ray enhancements, the gamma-rays have increased by a factor of $\sim 10$. We suggest that there is a newly formed accretion disk associated with UV emission, and the enhanced gamma-rays are produced via inverse Compton scattering between the UV emission from the disk and pulsar wind. At the same time, some fraction of the pulsar wind is stopped by the intrabinary shock, resulting in an increase in X-ray radiation. The accretion disk has been evaporated because of the gamma-rays from the pulsar magnetosphere, smearing out the pulsed radio emission even though the rotation-powered MSP is still active.

As Figure 1 indicates, the gamma-ray emission has brightened by a factor of 10 on a timescale of several days. In the inverse Compton scenario discussed in Section 3, the brightening timescale seen by Fermi corresponds to a migration timescale of the disk down to $r \sim 10^{8-9} \mathrm{~cm}$, where the inverse Compton scattering process is more efficient with a shorter mean free path than the process occurring at the outer part of the disk, $R \sim 10^{10-11} \mathrm{~cm}$. If one assumes a standard disk model, one can see that the migration timescale of the disk from $R \sim 10^{10} \mathrm{~cm}$ to $R \sim 10^{8-9} \mathrm{~cm}$ is of the order of several to 10 days; that is, $\tau_{m i} \sim R / v_{R} \sim 9 \alpha_{0.1}^{-4 / 5} M_{16}^{-3 / 10} R_{10}^{3 / 4}$ days, where $v_{R}$ is the radial velocity of the disk and $R_{10}=\left(R / 10^{10} \mathrm{~cm}\right)$.
Because UV/optical emissions are originated from the accretion disk, their variability timescale $\tau_{\mathrm{UV} / \mathrm{opt}} \sim 100 \mathrm{~s}$ may correspond to the Keplerian motion at the emission region. The standard disk model implies that UV/optical emissions with the observed luminosity level are mainly from the axial distance $R \sim 10^{9-10} \mathrm{~cm}$, corresponding to an orbital period of $\sim 160\left(R / 5 \times 10^{9}\right)^{3 / 2} \mathrm{~s}$, which is consistent with the observed timescale.

In the X-ray energy bands, the origin of the timescale $\tau_{X} \sim 50-100 \mathrm{~s}$ of the observed variability is more speculative with the shock emission model discussed in Section 3. But one may consider that the observed variability is caused by either perturbation of shock front due to clumpy stellar wind or wind speed variation $\left(v_{w} \sim 10^{8} \mathrm{~cm} \mathrm{~s}^{-1}\right)$, or sound propagation $\left(c_{s} \sim 10^{8} \mathrm{~cm} \mathrm{~s}^{-1}\right)$ in the shock front $\left(R_{s} \sim 5 \times 10^{10} \mathrm{~cm}\right)$. A more detailed consideration will be required to identify the origin of the X-ray variability.

J.T. and K.S.C. are supported by a GRF grant from the HK Government under HKU7009 11P. A.K.H.K. is supported by the National Science Council of the Republic of China (Taiwan) through grants NSC100-2628-M-007-002MY3, NSC100-2923-M-007-001-MY3, and NSC101-2119M-008-007-MY3. P.H.T. is supported by the National Science Council of the Republic of China (Taiwan) through grant NSC101-2112-M-007-022-MY3. C.Y.H. is supported by the National Research Foundation of Korea through grant 2011-0023383.

\section{REFERENCES}

Alpar, M. A., Cheng, A. F., Ruderman, M. A., \& Shaham, J. 1982, Natur, 300,728

Archibald, A. M., Kaspi, V. M., Bogdanov, S., et al. 2010, ApJ, 722, 88

Archibald, A. M., Kaspi, V. M., Hessels, J. W. T., et al. 2013, ApJ, submitted (arXiv:1311.5161)

Archibald, A. M., Stairs, I. H., Ransom, S. M., et al. 2009, Sci, 324, 1411

Becker, R. H., White, R. L., \& Helfand, D. J. 1995, ApJ, 450, 559

Blumenthal, G. R., \& Gould, R. J. 1970, RvMP, 42, 237

Bogdanov, S., Archibald, A. M., Hessels, J. W. T., et al. 2011, ApJ, 742, 97

Bond, H. E., White, R. L., Becker, R. H., \& O'Brien, M. S. 2002, PASP, 114,1359 
Campana, S., Colpi, M., Mereghetti, S., Stella, L., \& Tavani, M. 1998, A\&ARv, 8,279

Coroniti, F. V. 1990, ApJ, 349, 538

Eichler, D., \& Usov, V. 1993, ApJ, 402, 271

Frank, J., King, A., \& Raine, D. 2002, Accretion Power in Astrophysics (Cambridge: Cambridge Univ. Press)

Gaensler, B. M., Stappers, B. W., \& Getts, T. J. 1999, ApJL, 522, L117

Halpern, J. P., Gaidos, E., Sheffield, A., Price-Whelan, A. M., \& Bogdanov, S. 2013, ATel, 5514, 1

Homer, L., Szkody, P., Chen, B., et al. 2006, AJ, 131, 562

Jester, S., et al. 2005, AJ, 130, 873

Kong, A. K. H. 2013, ATel, 5515, 1

Kong, S. W., Cheng, K. S., \& Huang, Y. F. 2012, ApJ, 753, 127

Kong, S. W., Yu, Y. W., Huang, Y. F., \& Cheng, K. S. 2011, MNRAS, 416, 1067
Liang, K. R. 1999, Astrophysical Formulae (New York: Springer) Lyubarsky, Y., \& Kirk, J. G. 2001, ApJL, 547, L437

Nolan, P. L., Abdo, A. A., Ackermann, M., et al. 2012, ApJS, 199, 31

Papitto, A., Ferrigno, C., Bozzo, E., et al. 2013, Natur, 501, 517

Patruno, A., Archibald, A. M., Hessels, J. W. T., et al. 2014, ApJL, 781, L3

Ruderman, M., Shaham, J., \& Tavani, M. 1989, ApJ, 336, 507

Stappers, B. W., Archibald, A., Bassa, C., et al. 2013, ATel, 5513, 1

Takata, J., Cheng, K. S., \& Taam, R. E. 2012, ApJ, 745, 100

Takata, J., Cheng, K. S., \& Taam, R. E. 2010, ApJL, 723, L68

Tam, P. H. T., Hui, C. Y., Huang, R. H. H., et al. 2010, ApJL, 724, L207

Thorstensen, J. R., \& Armstrong, E. 2005, AJ, 130, 759

Wang, Y., Takata, J., \& Cheng, K. S. 2010, ApJ, 720, 178

Wang, Z., Archibald, A. M., Thorstensen, J. R., et al. 2009, ApJ, 703, 2017

Woudt, P. A., Warner, B., \& Pretorius, M. L. 2004, MNRAS, 351, 1015 